\title{
In vivo calcium imaging and Parkinson's disease
}

\author{
Tianwen Huang ${ }^{1,2} \&$ Qinyong $\mathrm{Ye}^{1,2^{*}}$ \\ ${ }^{1}$ Department of Neurology, Fujian Institute of Geriatrics, Fujian Medical University Union Hospital, Fuzhou 350001, China; \\ ${ }^{2}$ Key Laboratory of Brain Aging and Neurodegenerative Diseases, Fujian Key Laboratory of Molecular Neurology, \\ Fujian Medical University, Fuzhou 350001, China
}

Received November 21, 2016; accepted November 24; published online December 2, 2016

Citation: $\quad$ Huang, T., and Ye, Q. (2016). In vivo calcium imaging and Parkinson’s disease. Sci China Life Sci 59, 1338-1340. doi: 10.1007/s11427-016-0356-6

Brain function depends on patterns of synaptic input and neurocircuits, which are accompanied by transient changes in free intracellular calcium concentration (Resendez et al., 2015). A recent topic of interest has been the illumination of neuronal circuit activity by calcium imaging in vivo. As a new biological tool for recording behavioral signals in the animal brain, in vivo calcium imaging is performed to monitor neuronal activity based on intracellular calcium. In vivo calcium imaging is an advanced technique by which individual cells and/or even subcellular compartments can be observed in real time. Thus, it readily enables simultaneous recording of individual cells and subcellular compartments.

It is widely known that most depolarizing electrical signals rely on calcium influx to activate voltage-gated channels in the nervous system, which may then be further amplified by intracellular calcium stores, resulting in multiple physiological responses in neurons, such as synaptic transmission, neuronal plasticity, neuronal development, neuronal migration, and neurite outgrowth. On the other hand, transient intracellular elevation of calcium is involved in neuronal death or neurodegeneration (Ding et al., 2014). Thus, all of the evidence presented demonstrates that calcium signaling is obligatory for neuronal activities. Therefore, tracing dynamic changes of calcium in vivo will help us better understand neuronal function, and even brain function. However, the key to achieving high-quality dynamic calcium images is dependent on calcium indicators and imaging devices.

*Corresponding author (email: unionqyye@163.com)
There are two main classes of calcium indicators: chemical indicators and genetically encoded calcium indicators (GECI). The chemical indicators, including fura-2, indo-1, fluo-3, fluo-4, and calcium green-1, are small molecules that can chelate calcium ions, and are utilized for cytosolic calcium measurements in a wide variety of cells. However, these membrane-permeant forms of fluorometric calcium indicators are only able to satisfactorily load populations of neurons in cell culture or in slices of immature brain tissue. GECI are fluorescent proteins derived from green fluorescent protein (GFP) or its variants (such as circularly permuted GFP, YFP, and CFP), which fuse with the M13 domain of the myosin light chain kinase and calmodulin $(\mathrm{CaM})$, which is able to bind calcium. The genes encoding for GECI are easily transferred to cell lines. At the same time, it is possible to create a transgenic animal expressing the indicator in all cells or certain cellular subtypes. Because it showed high sensitivity and bright baseline fluorescence in a cellular environment, GCaMP6 has been used for calcium imaging of dendritic spines and putative postsynaptic sites in vivo (Sun et al., 2013; Chen et al., 2013).

Imaging devices are the other important factors in calcium imaging. The first real-time calcium imaging was performed in 1986 using intensified video cameras. Laser scanning in combination with calcium indicators is the latest technique to have made a breakthrough in imaging intracellular calcium. Moreover, two-photon calcium imaging further enhances this technique as a powerful tool for studying the function of calcium in the brain in vivo (Stosiek et al., 
2003). Using this technique, it is possible to analyze intact neuronal circuits in real time with the resolution of individual cells. However, the disadvantage of two-photon imaging is that the equipment must be fixed on the animal head, which greatly limits naturalistic behavior. In order to overcome limitations, new methods have been developed for freely moving animals. A new device, small and light enough to be carried by the animal, has been developed by combining mini-epifluorescence microscopes with gradient index (GRIN) lens microendoscopes and fiber photometry. The light weight and small size of the devices allows them to be coupled with a wide array of behavioral paradigms.

Considering the advantage of in vivo calcium imaging for evaluation of dynamic neuronal circuits, researchers have turned their attention to the basal ganglia circuits, which transform activity in the cerebrum into controlled motor learning, habit formation, and action selection. Two parallel striatal circuits regulate the effect of the basal ganglia on behavior. There are two subtypes of medium spiny neurons (MSNs): the direct pathway neurons express the dopamine D1 receptor (D1-MSNs), and the indirect pathway neurons express the dopamine $\mathrm{D} 2$ receptor (D2-MSNs).

In attempting to elucidate the functional details of the D1-MSNs and D2-MSNs in the dorsal striatum by using labeled molecular biological technique, Professor Lin (Barbera et al., 2016) ultimately determined how the MSNs acted to mediate behavior based on the GECI. The results further validated a dual circuit striatal model that concurrently activates both direct and indirect pathways in neurons during action initiation. At the same time, Professor Lin (Barbera et al., 2016) also found that by using neural cluster activities other than those in randomly selected subsets of single neurons or MSN populations, it was possible to predict behavioral states and locomotion velocities of mice. These findings were made possible by the application of calcium imaging in vivo and dependence on calcium indicators.

It is obvious that GECI are able to monitor the behavioral activities of populations of neurons and synapses. However, the transient nature of GECI is such that responses to calcium require online monitoring during behavioral activity. Furthermore, there may be differences in mouse activity depending on whether equipment is mounted on the head or the head is completely free. Therefore, the introduction of a new, permanent calcium molecular reporter that is able to record neural activity across the whole brain would be transformative, enabling focus on only marker neurons active during behavioral processes. Fortuitously, in 2015, Professor Schreiter's group (Fosque et al., 2015) designed a fluorescent sensor, the calcium-modulated photoactivatable ratiometric integrator (CaMPARI). CaMPARI has the characteristics of permanent, large-scale labeling of immediate early genes, which enables a temporally precise "activity snapshot" of a large tissue volume. Professor Schreiter
(Fosque et al., 2015) showed that when intracellular calcium is elevated, CaMPARI undergoes green-to-red conversion efficiently and irreversibly in response to a violet light shower in zebrafish, flies, head-fixed mice, and adult flies. Thus, the development of CaMPARI made it possible to efficiently study behavioral neuronal circuits in the entire brain.

Having summarized recent advances, we would like to share our insights about the application of in vivo calcium imaging in the study of mechanisms of disease or assessment of treatment, using the example of Parkinson's disease (PD).

Although the pathological changes have been characterized in different motion-related diseases and symptoms such as amyotrophic lateral sclerosis, epilepsy, monosymptomatic resting tremor, and PD (Fang et al., 2015a; Fang et al., 2015b; Cui et al., 2015; Zheng et al., 2015; Zhang et al., 2016), the in vivo identification of motor-induced active neurons and circuits is a fundamental challenge in understanding the neural basis of behavior. PD is a chronic, degenerative disorder of the central nervous system that mainly affects the motor system. The technique of in vivo calcium imaging is optimal for determining the activity of brain neurons and circuits in PD. The effects on movement and balance maintenance are mediated by D1-MSNs and D2-MSNs in striatal circuits. These activities may play opposing roles in promoting and inhibiting movement. Imbalance in their relative activities is thought to account for PD. However, the mechanisms by which neuronal circuits function in PD are unclear. Professor Lin's group has shown us how D1-MSNs and D2-MSNs in the dorsal striatum form spatially compact neural clusters, by providing information relevant to locomotion. Thus, further research on MSNs in PD can be performed by using GECI. The details of dysfunctional regulation in MSNs may be determined in PD. On the other hand, the in vivo utility of CaMPARI in the active neurons and circuits of the entire brain in PD animal models can be permanently observed upon illumination with violet light. Therefore, the information from MSNs of the dorsal striatum and from in vivo circuits will help us to understand the pathological processes in PD. It is well known that deep brain stimulation (DBS) is an effective treatment for PD. However, the mechanism by which DBS affects neuronal circuits is still unclear. The combination of in vivo calcium imaging technique and DBS in PD animal models will help us to identify the active neurons and circuits in the PD brain by observation of symptoms under different conditions.

In sum, the understanding of neurocircuits in vivo is fundamental to determining the neural basis of behavior. The use of GECI and CaMPARI to investigate locomotor dysfunction mediated by active neurons and neuronal circuits in $\mathrm{PD}$, through in vivo calcium imaging technique, may help us to understand the pathogenesis of PD. 
Compliance and ethics The author(s) declare that they have no conflict of interest.

Acknowledgements This work was supported by the National Natural Science Foundation of China (81271414).

Barbera, G., Liang, B., Zhang, L., Gerfen, C.R., Culurciello, E., Chen, R., Li, Y., and Lin, D.T. (2016). Spatially compact neural clusters in the dorsal striatum encode locomotion relevant information. Neuron 92, 202-213.

Chen, T.W., Wardill, T.J., Sun, Y., Pulver, S.R., Renninger, S.L., Baohan, A., Schreiter, E.R., Kerr, R.A., Orger, M.B., Jayaraman, V., Looger, L.L., Svoboda, K., and Kim, D.S. (2013). Ultrasensitive fluorescent proteins for imaging neuronal activity. Nature 499, 295-300.

Cui, Z.Q., Luan, G.M., Zhou, J., Zhai, F., Guan, Y.G., and Bao, M. (2015). Treatment of epilepsy with bipolar electro-coagulation: an analysis of cortical blood flow and histological change in temporal lobe. Chin Med J (Engl) 128, 210-215.

Ding, Y., Chang, C., Xie, L., Chen, Z., and Ai, H. (2014). Intense exercise can cause excessive apoptosis and synapse plasticity damage in rat hippocampus through $\mathrm{Ca}^{2+}$ overload and endoplasmic reticulum stress-induced apoptosis pathway. Chin Med J (Engl) 127, 3265-3271.

Fang, J., Liu, M.S., Guan, Y.Z., Cui, B., and Cui, L.Y. (2015a). Importance of sample size for the estimation of repeater $\mathrm{F}$ waves in amyotrophic lateral sclerosis. Chin Med J (Engl) 128, 515-519.
Fang, J., Cui, L.Y., Liu, M.S., Guan, Y.Z., Li, X.G., Cui, B., and Ding, Q.Y. (2015b). F Wave Study in Amyotrophic Lateral Sclerosis: Assessment of Segmental Motoneuronal Dysfunction. Chin Med J (Engl) $128,1738-1742$.

Fosque, B.F., Sun, Y., Dana, H., Yang, C.T., Ohyama, T., Tadross, M.R., Patel, R., Zlatic, M., Kim, D.S., Ahrens, M.B., Jayaraman, V., Looger, L.L., and Schreiter, E.R. (2015). Neural circuits. Labeling of active neural circuits in vivo with designed calcium integrators. Science 347 , $755-760$.

Resendez, S.L., and Stuber, G.D. (2015). In vivo calcium imaging to illuminate neurocircuit activity dynamics underlying naturalistic behavior. Neuropsychopharmacology 40, 238-239.

Sun, X.R., Badura, A., Pacheco, D.A., Lynch, L.A., Schneider, E.R., Taylor, M.P., Hogue, I.B., Enquist, L.W., Murthy, M., and Wang, S.S. (2013). Fast GCaMPs for improved tracking of neuronal activity. Nat Commun 4, 2170.

Stosiek, C., Garaschuk, O., Holthoff, K., and Konnerth, A. (2003). In vivo two-photon calcium imaging of neuronal networks. Proc Natl Acad Sci USA 100, 7319-7324.

Zhang, W., Zhang, L., Liang, B., Schroeder, D., Zhang, Z.W., Cox, G.A., Li, Y., and Lin, D.T. (2016). Hyperactive somatostatin interneurons contribute to excitotoxicity in neurodegenerative disorders. Nat Neurosci $19,557-559$.

Zheng, H.G., Zhang, R., Li, X., Li, F.F., Wang, Y.C., Wang, X.M., Lu, L.L., and Feng, T. (2015). Heterogeneity of Monosymptomatic Resting Tremor in a Prospective Study: Clinical Features, Electrophysiological Test, and Dopamine Transporter Positron Emission Tomography. Chin Med J (Engl) 128, 1765-1771.

Open Access This article is distributed under the terms of the Creative Commons Attribution License which permits any use, distribution, and reproduction in any medium, provided the original author(s) and source are credited. 